\title{
Type I Planet Migration in Weakly Magnetized Laminar Disks
}

\author{
Jérôme Guilet, Clément Baruteau and John C. B. Papaloizou
}

Department of Applied Mathematics and Theoretical Physics, University of Cambridge, Centre for Mathematical Sciences,

Wilberforce Road, Cambridge CB3 0WA

\begin{abstract}
Planet migration plays a crucial role in shaping planetary systems, and has therefore received a lot of attention in recent years in an effort to compare the statistical properties of observed exoplanets with the predictions of planet formation and migration theories. By modifying the propagation properties of the waves induced by the planet in the disk, the presence of a strong magnetic field can dramatically influence planet migration, in some cases reversing its direction. The more realistic case of a weaker magnetic field is less clear, although turbulent MHD simulations by Baruteau et al. (2011) suggest an effect on the corotation torque. Here, we present a study of the corotation torque in $2 \mathrm{D}$ laminar disks containing a toroidal magnetic field. We performed MHD simulations of the interaction between the magnetic field and the horseshoe motion of the gas, and found that this results in an additional corotation torque. This additional torque can be strong enough to reverse migration even for a field which pressure is only one percent of the thermal pressure. We speculate that this could lead to long range outward migration in the outer part of protoplanetary disks and may explain the observations by direct imaging of planets at several tens of AU from their star like the 4 planets system HR 8799.
\end{abstract}

Keywords. planetary systems: formation, planetary systems: protoplanetary disks, accretion, accretion disks, magnetic fields, MHD

\section{Introduction}

The gravitational interaction between planets and their parent protoplanetary disk gives rise to a torque exerted by the disk on a planet, which drives orbital migration. The hydrodynamical torque exerted on low mass planets (type I migration) has two components. The Lindblad differential torque due the spiral density waves launched at Lindblad resonances is negative and leads to fast inward migration. The corotation torque, due an exchange of angular momentum with the gas within its coorbital region, can be either positive or negative and may therefore in some circumstances slow down or reverse migration.

How is the picture modified by the expected presence of a magnetic field ? In the case of a strong azimuthal magnetic field that prevents horseshoe motion, the corotation torque is replaced by angular momentum carried away at magnetic resonances, where the rotational velocity relative to the planet is equal to the propagation speed of a slow MHD wave or an Alfvén wave (Terquem 2003, Fromang et al. 2005). In the intermediate case of a weak magnetic field, horseshoe motion can be expected to take place and give rise to a corotation torque. This has been confirmed by simulations of disks in which the magneto-rotational instability (MRI) drives magneto-hydrodynamical (MHD) turbulence (Baruteau et al. 2011, hereafter BFNM11). They showed the existence of horseshoe dynamics and an unsaturated corotation torque. They also found the existence of an additional corotation torque with moderate amplitude. In this proceeding, we investigate 
the physical origin and the properties of this additional corotation torque due to a weak magnetic field. The results presented in this proceeding are described in more details in Guilet et al. (2013).

\section{Physical and numerical setup}

\subsection{Disk model}

We consider a two-dimensional disk located at $z=0$ in a cylindrical polar coordinates system $(r, \varphi, z)$. We assume a locally isothermal equation of state, and a non-selfgravitating gas. The disk is laminar, and the effects of MHD turbulence are modeled by effective diffusion coefficients: a viscosity and a resistivity. The viscosity is parameterized by the usual $\alpha$ parameter : $\nu=\alpha c_{s}^{2} / \Omega_{K}$, where $c_{s}$ is the sound speed and $\Omega_{K}$ is the keplerian angular velocity. The value of the magnetic diffusivity $\eta$ is then determined by the assumed magnetic Prandtl number defined as $\mathcal{P}=\nu / \eta$, which is expected to be of order unity (Lesur \& Longaretti 2009). We assume a simple initial magnetic field geometry, with only a toroidal component $B_{\varphi}$. This is motivated by the fact that in sheared MHD turbulence the azimuthal component of the magnetic field tends to be the strongest one. Furthermore, we initialize the calculation with power law profiles for the surface density $\Sigma$, temperature $T$ and azimuthal magnetic field $B_{\varphi}$. The power law indices are defined as $b \equiv d \log B_{\varphi} / d \log r, p \equiv d \log \Sigma / d \log r$, and $q \equiv d \log T / d \log r$. The strength of the magnetic field is measured in terms of the plasma parameter $\beta$, which is defined as the ratio of the thermal to the magnetic pressure:

$$
\beta=P_{\mathrm{th}} / P_{\mathrm{mag}}=2 c_{s}^{2} / v_{A}^{2},
$$

where $v_{A} \equiv B / \sqrt{\mu_{0} \rho}$ is the Alfvén velocity, and $\mu_{0}$ is the vacuum permeability.

A planet is introduced in the disk at the beginning of the simulations and is held in a fixed circular orbit, at a radius $r_{p}$ and azimuth $\varphi_{p}=0$. We work in the frame rotating with the planet at angular frequency $\Omega_{p}$. The planet potential is smoothed over a softening length $\varepsilon=0.6 H\left(r_{p}\right)$. Except in Section 4, the torque calculations presented in this paper include the planet's Hill sphere. Finally, note that all the torques and torque density distributions presented in this paper are per unit mass of the planet.

\subsection{Normalization, fiducial parameters and migration regime}

We normalize the radius, surface density, time and magnetic field such that $r_{p}=1$, $\Sigma\left(r_{p}\right)=1, \Omega_{p}=1$ and $\mu_{0}=1$. We use the following fiducial parameters : a planet-toprimary mass ratio of $M_{p}=2 \times 10^{-5} M_{\star}$ (around 7 earth mass's for a Sun-like star), a disk aspect ratio $h=0.05$, a plasma parameter $\beta=100$, diffusion coefficients $\alpha=5.10^{-3}, \mathcal{P}=$ 1 , and a slope indice of the magnetic field $b=-1$. For $p$ and $q$, we consider the following fiducial disk model (Model 1 in BFNM11) : $p=-1 / 2$ and $q=-1$, corresponding to a uniform aspect ratio of the disk.

The fiducial value of the planet mass falls into the regime of type I migration, since $M_{p} /\left(M_{*} h^{3}\right)=0.16$. The relative strength of the magnetic field and horseshoe motion may be measured with the ratio of the Alfvén speed to the shear velocity at the separatrix of the horseshoe region:

$$
\frac{v_{A}}{v_{\varphi}\left(r_{p}-x_{s}\right)} \simeq 0.86 \sqrt{\frac{M_{*} h^{3}}{M_{p} \beta}} .
$$

The fiducial parameters give: $v_{A} / v_{\phi}\left(x_{s}\right) \simeq 0.21$. Since the Alfvén speed is significantly smaller than the shear velocity at the separatrix of the horseshoe region, the magnetic 
field is not expected to prevent horseshoe motion of the gas. If they were present, the magnetic resonances would lie within the horseshoe region. We therefore do not expect to see magnetic resonances but rather horseshoe motion modified by the presence of the magnetic field.

\subsection{Numerical method}

We use two different MHD codes : the finite volume code RAMSES (Fromang et al. 2006), and the finite difference code NIRVANA (Ziegler \& Yorke 1997). The numerical domain extends around the planet location in the range $r \in[0.5,2]$, and $\phi \in[-\pi, \pi]$. Wave-killing zones are used near the grid's inner edge $(r \in[0.5,0.65])$ and outer edge $(r \in[1.7,2])$ in order to avoid spurious reflections of the planet's wakes. Our default grid resolution is such that the half-width of the horseshoe region is resolved by about 8 cells for our fiducial set of parameters. We found that this resolution is good enough to obtain converged results.

\section{An additional corotation torque of magnetic origin}

A global view of a simulation with the fiducial parameters is represented in Figure 1. The density perturbation (left panel) shows two distinct features: the usual wake (i.e. the spiral density waves launched in the inner and the outer disk), and an underdense region localized around the planet's orbital radius. This second feature is due to the horseshoe dynamics in the presence of a magnetic field and will be described further below. The magnetic field perturbation is mostly located near the corotation, indicating that the main effect of the relatively weak magnetic field considered here is on the horseshoe dynamics.

In Figure 2 we show a close up view of the horseshoe region, and compare simulations using our fiducial magnetization $\beta=100$ (upper panels) with simulations using an extremely weak magnetization $\left(\beta=10^{8}\right.$, lower panels) where the magnetic field is effectively passive. The horseshoe motion of the gas concentrates the magnetic field on the dowstream separatrices of the horseshoe region, resulting in a similar magnetic configuration for our fiducial magnetic field and a passive one (left panels). This is the result of a competition between the advection due to horseshoe motion and diffusion by the
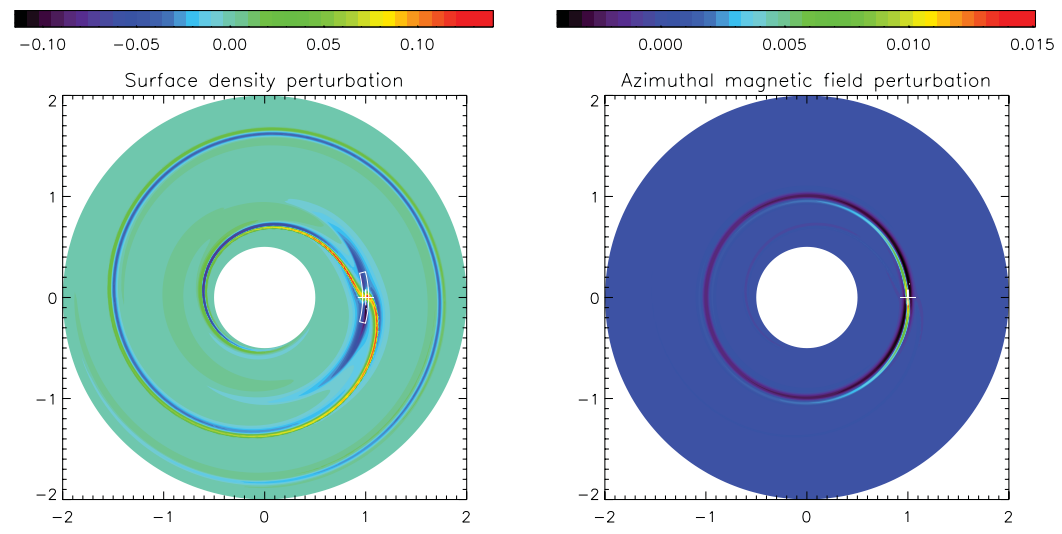

Figure 1. Global view of a simulation with the fiducial parameters. The left panel shows the surface density perturbation, while the right panel shows the perturbation of the azimuthal component of the magnetic field. The location of the planet is shown with a white + sign at $r=1, \varphi=0$. The extent of the region around the planet represented in Figures 2 is shown with a white sector in the left panel. 

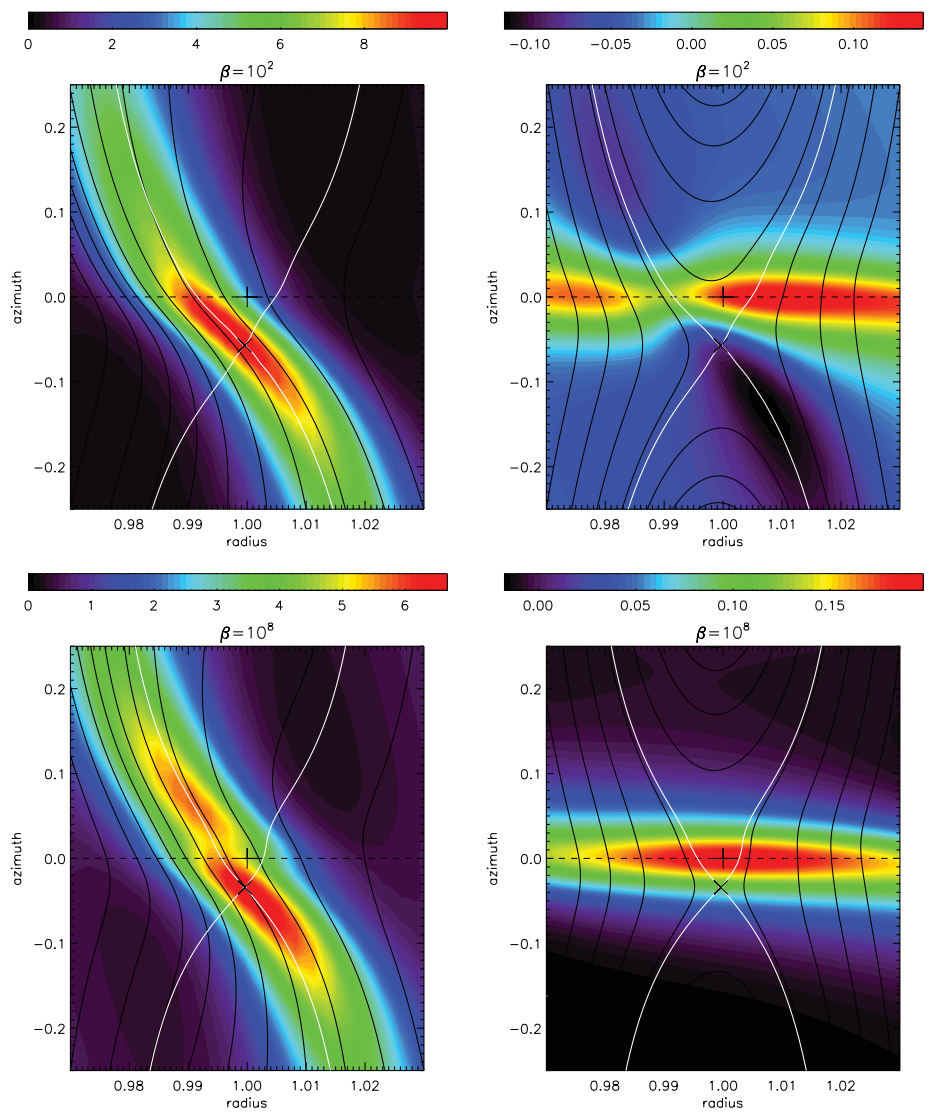

Figure 2. Comparison of simulations using our fiducial magnetization $\beta=100$ (upper panels) with simulations using an extremely weak magnetization $\left(\beta=10^{8}\right.$, lower panels) where the magnetic field is effectively passive. Left panels: the color contours show the magnetic energy normalized by its initial value. The black lines represent magnetic field lines. Right panels: the color contours show the density perturbations. The black lines represent the streamlines. In all panels, the white lines represent the separatrices delimiting the horseshoe region, the black cross shows the position of the stagnation point at the intersection of the two separatrices. The planet location is depicted by a black + sign at $r=1, \varphi=0$.

turbulent resistivity. The increased magnetic pressure near the downstream separatrices creates an underdensity in the magnetized case in order to ensure approximate pressure balance (right panels). Importantly an azimuthal asymmetry with respect to the planet's location is clearly visible: the stagnation point is located at negative azimuth in this model, and the underdensity as well as the increased magnetic pressure are preferentially at negative azimuth. The asymmetry of the density distribution results in a net torque exerted on the planet, which time evolution is shown in Figure 3. The magnetic field gives rise to a positive additional torque (which we term MHD torque excess), which is larger than the hydrodynamical torque. As a result a positive total torque is obtained, corresponding to outward migration.

The properties of the MHD torque excess were characterized by systematically varying the slope indices of the density, temperature and magnetic field, the viscosity and resistivity as well as the magnetic field strength. We found that the MHD torque excess has an opposite sign to that of the azimuth of the stagnation point. The sign of the stagnation point azimuth $\varphi_{s}$ is independent of the magnetic field and is governed by the density 


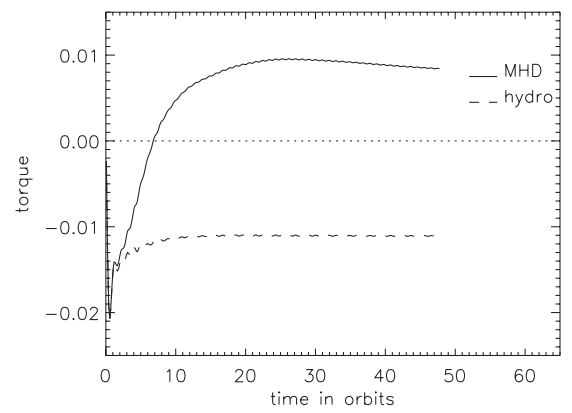

Figure 3. Time evolution of the torque exerted on the planet in our fiducial model, with and without a magnetic field (full and dashed lines respectively).
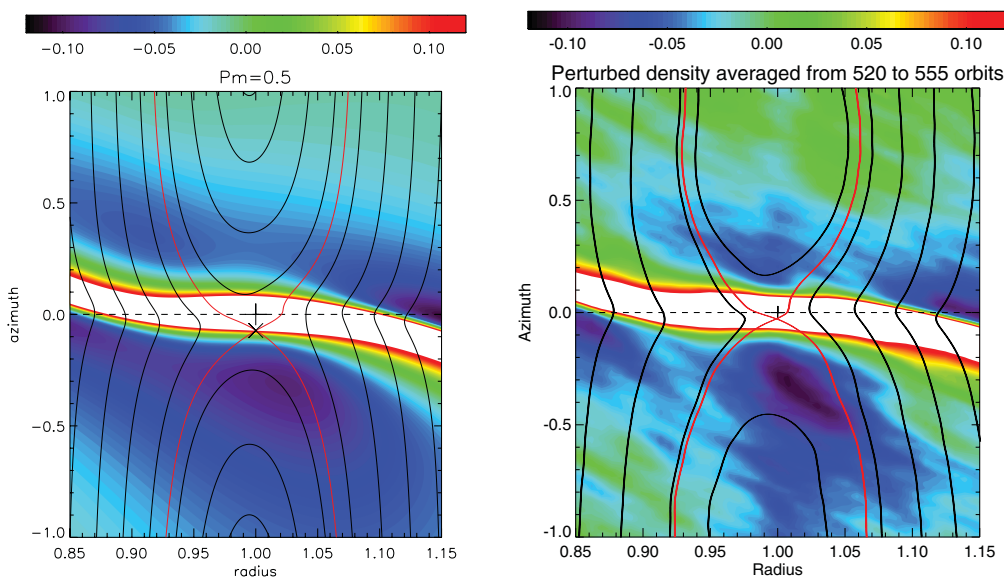

Figure 4. Comparison of the density perturbation obtained in our 2D laminar simulations (left panel) with that of the 3D turbulent simulations of BFNM11. Both simulations clearly show a negative density perturbation near the downstream separatrix at negative azimuth.

and temperature profiles in the following way (fit obtained from hydrodynamical simulations): $\operatorname{sign}\left(\varphi_{s}\right)=\operatorname{sign}(1.3 q-p-0.6)$. The MHD torque excess is therefore expected to be positive for profiles typical of protoplanetary disks. On the other hand, the magnitude of the stagnation point azimuth with respect to the azimuth of the planet is increased by presence of the magnetic field. The amplitude of the MHD torque excess increases strongly when the magnetic field strength is increased or when the turbulent resistivity is decreased (and it is roughly independent of the gradients of density, temperature and magnetic field). This dependence on the resistivity is due to the fact that a lower resistivity allows more concentration of magnetic flux on the downstream separatrices.

\section{Comparison with 3D MHD simulations of disks with turbulence due to the MRI}

In order to check how well a $3 \mathrm{D}$ turbulent situation can be described by $2 \mathrm{D}$ simulations where the effects of turbulence are modeled by diffusion coefficients, we compared our results with the 3D MHD simulations of disks performed by BFNM11, where the MRI operates throughout the whole disk. For this purpose, we ran $2 \mathrm{D}$ simulations using the same parameters as in BFNM11, in particular with a viscosity and magnetic field strength set to $\alpha=3.10^{-2}$, and $\beta=50$ and the same disk model as in the rest of this proceeding 

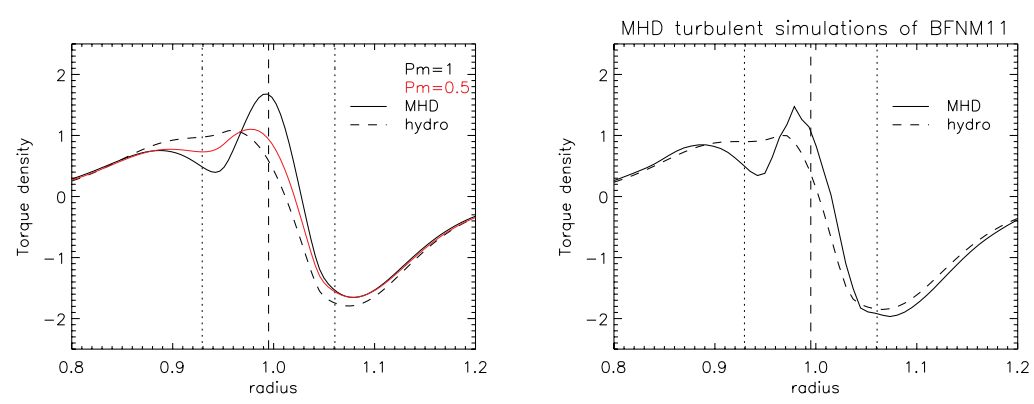

Figure 5. Comparison of the torque density distribution obtained in our 2D laminar simulations with that of the 3D turbulent simulations of BFNM11. Hydrodynamic simulations are shown with dashed lines, while their MHD counterpart are shown with full lines. Furthermore, we considered 2 values of the magnetic Prandtl number (setting the resistivity): $P_{m}=1$ (black lines) and $P_{m}=0.5$ (red lines). The torques and torque densities are calculated excluding the Hill radius for consistency with BFNM11.

(the magnetic Prandtl was not measured in BFNM11, so two values are considered here $\mathcal{P}=0.5$ and $\mathcal{P}=1$ ). The density perturbation is displayed in Figure 4: the underdense region located near the downstream separatrix at negative azimuth is clearly visible in both the 2D laminar simulation (left panel) and the $3 \mathrm{D}$ turbulent simulation (right panel). The torque density distribution, shown in Figure 5, is also very similar in the turbulent and laminar runs. This gives confidence that the same physical process is at work in both situations.

\section{Conclusion}

We showed that a weak toroidal magnetic field such as created by the magnetorotational instability can give rise to a significant additional torque on a low mass planet. This torque excess is positive for disk profiles typical of protoplanetary disk and can be strong enough to reverse the direction of migration (Guilet et al. 2013). A particularly interesting consequence is that an outward migration is then possible in the radiatively efficient outer parts of protoplanetary disks, where the hydrodynamical torque would otherwise lead to inward migration. We speculate that this could lead to a long range outward migration in the outer part of protoplanetary disks and may explain the observations by direct imaging of planets at several tens of AU from their star like the 4 planets system HR 8799 (Marois et al. 2008).

Future research should study the effect on planet migration of a vertical magnetic field, which is expected to be present in protoplanetary disks (Ferreira et al. 2006, Muto et al. 2008, Guilet \& Ogilvie 2012, 2013).

\section{References}

Baruteau C., Fromang S., Nelson R. P., \& Masset F., 2011, A\&A, 533, A84

Ferreira J., Dougados C., \& Cabrit S., 2006, A\&A, 453, 785

Fromang S., Hennebelle P., \& Teyssier R., 2006, A\&A, 457, 371

Fromang S., Terquem C., \& Nelson R. P., 2005, MNRAS, 363, 943

Guilet J., Baruteau C., \& Paploizou J. C. B., 2013, MNRAS, 430, 1764

Guilet J. \& Ogilvie G. I., 2012, MNRAS, 424, 2097

Guilet J. \& Ogilvie G. I., 2013, MNRAS, 430, 822

Lesur G. \& Longaretti P.-Y., 2009, A\&A, 504, 309 
Marois C., Macintosh B., Barman T., Zuckerman B., Song I., Patience J., Lafrenière D., \& Doyon R., 2008, Science, 322, 1348

Muto T., Machida M. N., \& Inutsuka S.-i., 2008, ApJ, 679, 813

Terquem C. E. J. M. L. J., 2003, MNRAS, 341, 1157

Ziegler U. \& Yorke H. W., 1997, Computer Physics Communications, 101, 54 